\title{
Allograft inflammatory factor-1 in the pathogenesis of bleomycin- induced acute lung injury
}

\author{
Hidetake Nagahara, Aihiro Yamamoto, Takahiro Seno, Hiroshi Obayashi, Takashi Kida, \\ Amane Nakabayashi, Yuji Kukida, Kazuki Fujioka, Wataru Fujii, Ken Murakami, \\ Masataka Kohno, Yutaka Kawahito*
}

Inflammation and Immunology, Graduate School of Medical Science, Kyoto Prefectural University of Medicine, Kyoto, Japan.

\begin{abstract}
Summary Allograft inflammatory factor-1 (AIF-1) is a protein expressed by macrophages infiltrating the area around the coronary arteries of rats with an ectopic cardiac allograft. Some studies have shown that expression of AIF-1 increased in a mouse model of trinitrobenzene sulfonic acid-induced acute colitis and in acute cellular rejection of human cardiac allografts. These results suggest that AIF-1 is related to acute inflammation. The current study used bleomycininduced acute lung injury to analyze the expression of AIF-1 and to examine its function in acute lung injury. Results showed that AIF-1 was significantly expressed in lung macrophages and increased in bronchoalveolar lavage fluid from mice with bleomycin-induced acute lung injury in comparison to control mice. Recombinant AIF-1 increased the production of IL-6 and TNF- $\alpha$ from RAW264.7 (a mouse macrophage cell line) and primary lung fibroblasts, and it also increased the production of KC (CXCL1) from lung fibroblasts. These results suggest that AIF-1 plays an important role in the mechanism underlying acute lung injury.
\end{abstract}

Keywords: Allograft inflammatory factor 1, AIF-1, acute lung injury, IL-6, TNF- $\alpha$, KC

\section{Introduction}

Acute lung injury (ALI) and its severe manifestation, acute respiratory distress syndrome (ARDS), are lifethreatening inflammatory conditions that involve acute respiratory failure $(1,2)$. Lung injury causes the release of pro-inflammatory cytokines and the recruitment of neutrophils in lung tissue (3-6). Activation of cytokines and infiltrating neutrophils damage the alveolarcapillary barrier and lead to subsequent pulmonary fibrosis (7-9). Despite recent advances in studies of the pathogenesis of ALI and ARDS, the molecular mechanism that initiates ALI and ARDS has not been elucidated in detail.

Allograft inflammatory factor 1 (AIF-1) is a 17-

Released online in J-STAGE as advance publication February 25, 2016.

*Address correspondence to:

Dr. Yutaka Kawahito, Inflammation and Immunology, Graduate School of Medical Science, Kyoto Prefectural University of Medicine, 465, Kajii-cho, Kawaramachi-Hirokoji, Kamigyoku, Kyoto 602-8566, Japan.

E-mail: kawahity@koto.kpu-m.ac.jp
$\mathrm{kDa} \mathrm{Ca}^{2+}$-binding EF-hand intracellular protein that is encoded by the HLA class III genomic region (1012). AIF-1 was originally cloned from activated macrophages in atherosclerotic allogenic heart grafts undergoing chronic immune rejection in a rat (13). Although the expression of AIF-1 has been noted in various tissues such as the testes, spleen, lymph nodes, lungs, thymus, and synovium, the detailed role of AIF-1 remains unclear $(10,14-16)$.

The expression of AIF-1 increased two-fold in acutely rejected liver allografts in comparison to accepted liver grafts (17). Studies have shown that expression of AIF-1 increased in a model of trinitrobenzene sulfonic acid-induced acute colitis and in acute cellular rejection of cardiac allografts $(18,19)$. In vitro, CRL2192 (a rat macrophage cell line) expressed a certain level of endogenous AIF-1, and this expression was enhanced with the pro-inflammatory cytokines IL-1 $\beta$ and TNF- $\alpha$ (20). AIF-1-transfected CRL-2192 cells had enhanced levels of monocyte chemoattractant protein 1 and enhanced cell migration (20). AIF-1transfected RAW 264.7 cells had enhanced production of the inflammatory cytokines IL-6 and IL-12 as a result of stimulation with a lipopolysaccharide (21). These 
findings suggest that AIF-1 plays a crucial role in the mechanism of inflammation. The present study used a model of bleomycin-induced lung injury to examine the expression and function of AIF-1 in ALI.

\section{Materials and Methods}

\subsection{Bleomycin-induced ALI}

C57BL/6 mice (8-10 weeks of age) were purchased from Shimizu Laboratory Supplies Co., Ltd. Mice were anesthetized by intraperitoneal administration of pentobarbital. Thirty $\mu \mathrm{L}$ of bleomycin hydrochloride (Nippon Kayaku Co, Tokyo, Japan) solution containing $2.15 \mathrm{U} / \mathrm{kg}$ of bleomycin dissolved in sterile saline was injected directly into the trachea using a 0.9mm feeding needle (KN-348 Natsume Seisakusho Co., Tokyo, Japan). Control mice received the same volume of sterile saline alone. Mice were sacrificed on Day 1 by intraperitoneal administration of excess pentobarbital $(120-150 \mathrm{mg} / \mathrm{kg})$. Mice were euthanized if their body weight fell below $80 \%$ of that measured at the baseline. The study was approved by the Animal Research Committee, Graduate School of Medical Science, Kyoto Prefectural University of Medicine.

\subsection{Histology}

The lungs were excised on Day 1 after intratracheal administration of bleomycin and immediately fixed with $4 \%$ paraformaldehyde. The samples were then embedded in paraffin and stained with hematoxylin and eosin (H\&E) method. Images were acquired using a DMBA210 microscope (Shimadzu Rika, Tokyo, Japan) equipped with Motic Images Plus2.2s software (Shimadzu Rika).

\subsection{Immunohistochemical analysis}

The lungs were excised on Day 1 after intratracheal administration of bleomycin and immediately fixed with $4 \%$ paraformaldehyde. Paraffin-embedded sections were deparaffinized in xylol and rehydrated through a graded series of ethanol solutions. Endogenous peroxidase was blocked by incubation in $3 \% \mathrm{H}_{2} \mathrm{O}_{2}$ for $30 \mathrm{~min}$. Sections were stained with anti-AIF-1 antibody (Sigma) (1:150), anti-F4/80 antibody (Serotec) (1:100) or normal goat IgG isotype control overnight followed by MAX-PO (Nitirei Bioscience) for $30 \mathrm{~min}$. Finally, sections were stained with diaminobenzidine (Nitirei Bioscience). All sections were counterstained with hematoxylin for $2 \mathrm{~min}$.

\subsection{Bronchoalveolar lavage fluid (BALF)}

BALF analyses were performed on Day 1 after intratracheal administration of bleomycin as previously described (22). Briefly, the lungs and trachea were exposed and a 20-gauge intravenous catheter was inserted into the trachea. A total of $1 \mathrm{~mL}$ of PBS was instilled three times and withdrawn from the lungs via an intratracheal cannula. More than $95 \%$ of the fluid was recovered as BALF, which was then centrifuged at $1,000 \mathrm{rpm}$ for $5 \mathrm{~min}$ at $4^{\circ} \mathrm{C}$. The supernatant was collected and stored at $-80^{\circ} \mathrm{C}$ for use in ELISA. The levels of AIF-1, IL- 6 , TNF- $\alpha$, and KC in the BALF were measured using commercial ELISA kits (an AIF-1 ELISA kit; USCN Lifescience, an IL-6 ELISA kit and a TNF- $\alpha$ ELISA kit; eBioscience, an KC ELISA kit; R\&D Systems), according to the manufacturer's instructions. The optical density was measured at $450 \mathrm{~nm}$ using a SoftMaxPro40 plate reader. Each measurement was determined in three separate experiments on BALF. The total number of cells in BALF was counted using a Fuchs-Rosenthal hemocytometer (ERMA Inc., Tokyo, Japan). The BALF solution was placed in a cytospin (Cytospin 2; Shandon Inc., Pittsburgh, PA, USA), centrifuged at $700 \mathrm{rpm}$ for 10 minutes, and stained with Diff-Quick (Sysmex, Kobe, Japan). The number of total cells, neutrophils, and macrophages were counted. At least 200 cells per slide were evaluated on the basis of morphological criteria using a light microscope.

\subsection{Isolation of primary lung fibroblasts}

Primary lung fibroblasts were isolated from C57BL/6 mice and cultured as previously described $(23,24)$. Briefly, normal C57BL/6 mice (8-10 weeks-of-age) were sacrificed by intraperitoneal administration of excess pentobarbital and lung tissue was promptly collected, washed with phosphate buffered saline (PBS), and cut into approximately $2.0-\mathrm{mm}^{3}$ blocks. The blocks were seeded onto the bottom of a culture flask containing RPMI 1640 medium (Wako, Osaka, Japan) supplemented with $10 \% \mathrm{FBS}, 100 \mathrm{U} / \mathrm{mL}$ penicillin, and $100 \mathrm{mg} / \mathrm{mL}$ streptomycin, and the fibroblasts were cultured at $37^{\circ} \mathrm{C}$ in a $5 \% \mathrm{CO}_{2}$ incubator. The medium was changed every 2-3 days. Lung fibroblasts from three to five passages were used for all experiments.

\subsection{Cell culture}

A mouse macrophage cell line, RAW 264.7, was obtained from the RIKEN cell bank (Tsukuba, Japan). RAW264.7 cells were seeded in 12-well plates $\left(5 \times 10^{5}\right.$ cells/well) and primary lung fibroblasts were seeded in 12-well plates $\left(2 \times 10^{5}\right.$ cells/well $)$ in RPMI-1640 supplemented with $10 \%$ FBS for $24 \mathrm{~h}$. Media were replaced with serum-free RPMI-1640 and the cells were serum-starved overnight. Next, the cells were stimulated for $24 \mathrm{~h}$ by addition of RPMI-1640 supplemented with various concentrations of recombinant AIF-1 (CUSABIO, endotoxin removed) or a lipopolysaccharide (LPS) (Sigma). The cell culture supernatant was collected and stored at $-80^{\circ} \mathrm{C}$ until required. The levels of IL- 6 , TNF- $\alpha$ 
and $\mathrm{KC}$ in the cell culture supernatant were measured using commercial ELISA kits. The optical density was measured at $450 \mathrm{~nm}$ using a SoftMaxPro40 plate reader. Each measurement was determined in three separate experiments.

\subsection{Quantitative real-time RT-PCR analyses}

On Day 1 after intratracheal administration of saline or bleomycin, cells were seeded at a concentration of $1 \times$ $10^{5}$ cells/well in 24-well plates and cells were incubated in RPMI-1640 supplemented with 10\% FBS for $24 \mathrm{~h}$. The next day, the medium was washed with PBS three times and only cells adhering to the bottom of plates (alveolar macrophages) were collected. Total RNA was obtained from the macrophages with an RNeasy Plus Mini Kit (Qiagen). Quantitative real time RT-PCR was performed on StepOne ${ }^{\mathrm{TM}}$ \& StepOnePlus ${ }^{\mathrm{TM}}$ Real-Time PCR Systems (Life Technologies) using the Thunderbird Probe qPCR Mix (TOYOBO). The following TaqMan expression assays were used: Mm00479862_g1 (AIF-1), Mm99999915_g1 (GAPDH). Results in duplicate were normalized to GAPDH expression. Data are expressed as the mean -fold change relative to control samples.

\subsection{Statistical analysis}

Results are expressed as either the mean \pm SEM or the median as appropriate. All values were analyzed with a Mann-Whitney $U$ test. $p$ values $<0.05$ were considered statistically significant.

\section{Results}

\subsection{Inflammatory cells increased in lung tissue and $B A L F$ from mice with bleomycin-induced ALI}

To estimate the degree of inflammation in lung tissue after treatment with saline or bleomycin, tissue samples were stained with H\&E. Lung tissue from mice exposed to bleomycin on Day 1 displayed severe inflammation in comparison to tissue from the control group (Figures 1A-1D). BALF from mice exposed to bleomycin showed that the total cell number and the number of neutrophils and macrophages on Day 1 increased in comparison to numbers in the control group (Figure 1E).

3.2. AIF-1 is expressed in lung tissue from mice with bleomycin-induced ALI

To estimate the expression of AIF-1 in lung tissue on Day 1 after treatment with saline or bleomycin, samples were stained with an anti-AIF-1 Ab or anti-F4/80 Ab. Cells expressing AIF-1 were not evident in lung tissue after treatment with saline (Figures $2 \mathrm{~A}$ and $2 \mathrm{E}$ ). In contrast, invasive cells (arrow head) that were mostly

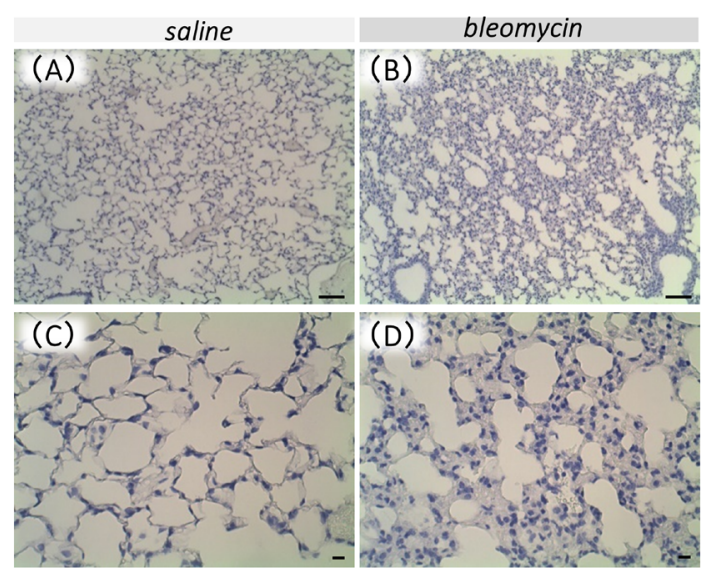

(E) BALF
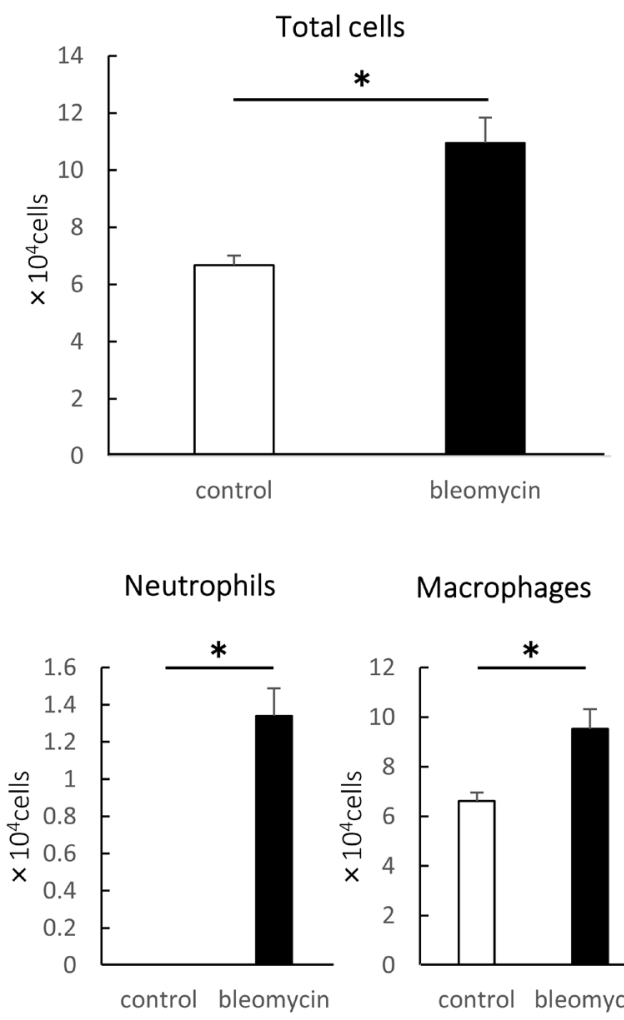

Macrophages

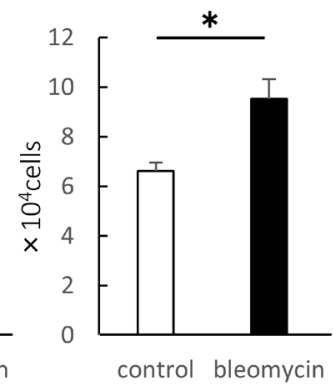

Figure 1. Infiltration of inflammatory cells in lung tissue and BALF from mice with bleomycin-induced acute lung injury. (A-D) H\&E staining. (E) Cell number in BALF $(n=$ 4 mice per group). (A, C) Lung tissue from control on Day 1. (B, D) Lung tissue from mice with bleomycin-induced acute lung injury on Day 1. (E) Total cell number, the number of neutrophils and macrophages in BALF on Day 1. (A, B) magnification, $\times 100$; Scale bars, $100 \mu \mathrm{m}$. (C, D) magnification, $\times 400$; Scale bars, $10 \mu \mathrm{m}$. Each bar represents the mean \pm SE. ${ }^{*} p<0.05$.

round with round nuclei and that expressed AIF-1 were evident in lung tissue after treatment with bleomycin (Figures 2B and 2F). Invasive macrophages (arrow head) that were mostly round with round nuclei and that expressed F4/80 were evident in lung tissue after treatment with bleomycin (Figures 2D and 2H). These results indicate that AIF-1 was expressed in invasive macrophages. Immunostaining of lung tissue with normal IgG was completely negative after treatment with bleomycin (Figures 2C and 2G). 

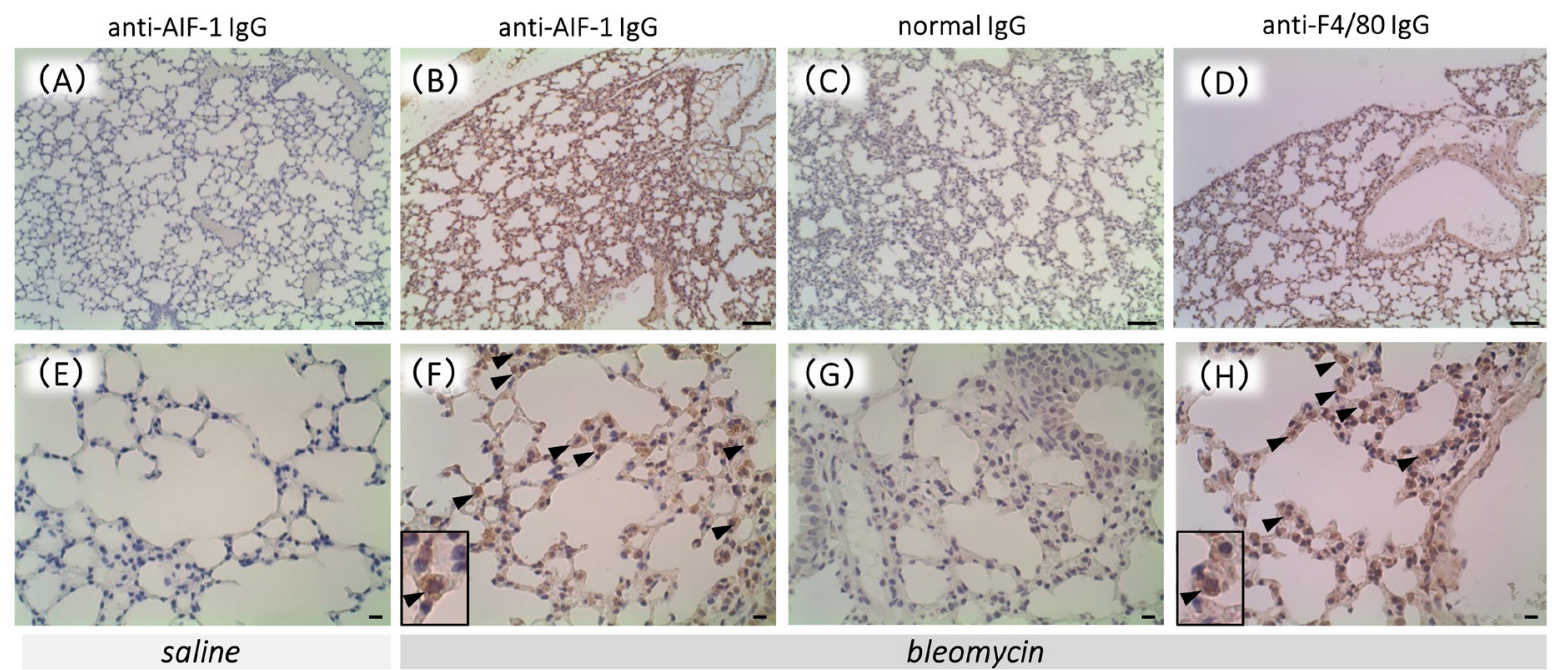

Figure 2. Expression of AIF-1 in lung tissue from mice with bleomycin-induced acute lung injury. (A-H) Immunohistochemical analysis. (A, E) Lung tissue on Day 1 after treatment with saline. (B-D, F-H) Lung tissue on Day 1 after treatment with bleomycin. (A, E) Immunostaining with an anti-AIF-1 antibody. (B, F) Immunostaining with an anti-AIF-1 antibody (brown) (arrow head). (C, G) Immunostaining with normal IgG. (D, H) Immunostaining with an anti-F4/80 antibody (brown) (arrow head). (A-D) magnification, $\times 100$; Scale bars, $100 \mu \mathrm{m}$. (E-H) magnification, $\times 400$; Scale bars, $10 \mu \mathrm{m}$.
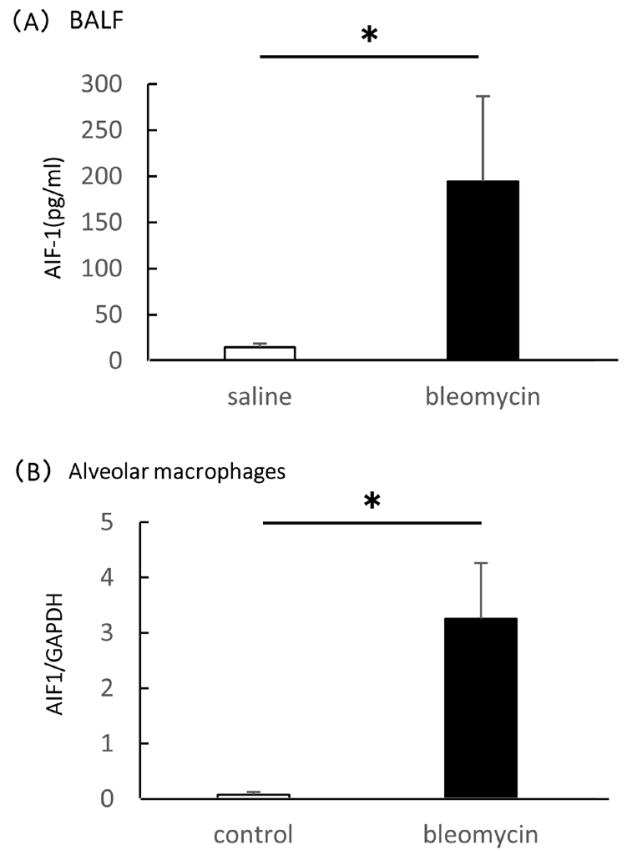

Figure 3. Expression of AIF-1 in BALF and alveolar macrophages in BALF from mice with bleomycin-induced acute lung injury. (A) AIF-1 ELISA $(n=4$ mice per group). (B) AIF-1 real-time PCR ( $n=4$ mice per group). (A) AIF-1 level in BALF on Day 1. (B) The level of AIF-1 mRNA in alveolar macrophages in BALF on Day 1. Each bar represents the mean \pm SE. ${ }^{*} p<0.05$

\subsection{AIF-1 is expressed in BALF and alveolar macrophages in BALF from mice with bleomycin-induced ALI}

To examine the expression of AIF-1 in BALF on Day 1 after treatment with saline or bleomycin, the concentration of AIF-1 was measured using ELISA. In BALF, the level of AIF-1 expression was significantly increased in comparison to that in the control group
(Figure 3A). The expression of AIF-1 was examined in alveolar macrophages from BALF. Real-time PCR analysis indicated that the level of AIF-1 mRNA was significantly increased in alveolar macrophages from BALF on Day 1 after intratracheal administration of bleomycin in comparison to administration of saline (Figure 3B).

\subsection{AIF-1 stimulates RAW264.7 cells and lung fibroblasts to secrete IL-6 and TNF- $\alpha$}

To estimate the levels of inflammatory cytokines in BALF after treatment with saline or bleomycin, the levels of IL- 6 and TNF- $\alpha$ were measured. BALF from mice exposed to bleomycin on Day 1 displayed significantly increased levels of IL- 6 and TNF- $\alpha$ in comparison to levels in the control group (Figures 3A and 4B). The effect of recombinant AIF-1 on cytokine secretion by RAW264.7 cells and lung fibroblasts was examined in vitro. Cultured RAW264.7 cells and lung fibroblasts were stimulated for $24 \mathrm{~h}$ with various concentrations of recombinant AIF-1 or LPS. The levels of IL- 6 and TNF- $\alpha$ secreted by AIF-1 (10 ng/mL-1 $\mu \mathrm{g} /$ $\mathrm{mL}$ )-treated RAW264.7 cells were significantly higher than those secreted by control cells, and this effect was dose-dependent (Figures 4C and 4D). The levels of IL-6 and TNF- $\alpha$ secreted by AIF-1-treated lung fibroblasts were also significantly higher than those secreted by control cells, and this effect was also dose-dependent (Figures 4E and 4F).

\subsection{AIF-1 stimulates lung fibroblasts to secrete $K C$}

The number of neutrophils in BALF increased on Day 1 after mice were exposed to bleomycin (Figure 1E). The 
(A)
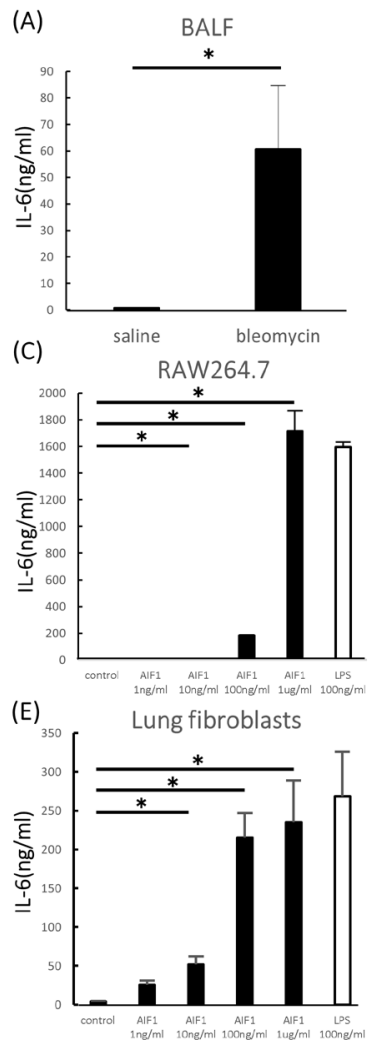

(B)
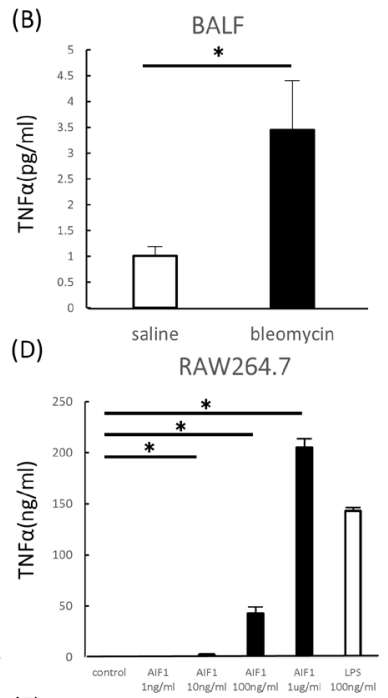

(F)

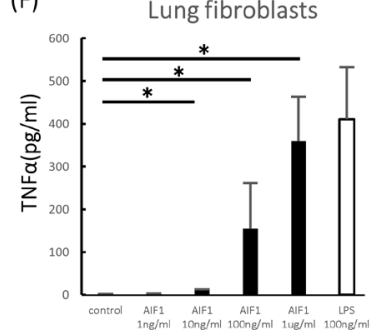

Figure 4. The levels of IL-6 and TNF- $\alpha$ in BALF from mice with bleomycin-induced acute lung injury and the levels of IL-6 and TNF- $\alpha$ secreted from RAW264.7 cells and lung fibroblasts stimulated by recombinant AIF-1. (A, B) IL-6 and TNF- $\alpha$ levels in BALF on Day 1 after intratracheal administration of saline or bleomycin. ( $n=4$ mice per group). (C-F) IL-6 and TNF- $\alpha$ levels in supernatant $(n=5$ mice per group). The levels of IL- 6 and TNF- $\alpha$ in the supernatant from cultured RAW264.7 cells and lung fibroblasts were measured after cells were exposed to various concentrations of rAIF-1 for $24 \mathrm{~h}$. Each bar represents the mean \pm SE. ${ }^{*} p<0.05$.

concentration of $\mathrm{KC}$, which is involved in chemotaxis and cell activation of neutrophils, was measured next. BALF from mice exposed to bleomycin on Day 1 had a significantly increased level of $\mathrm{KC}$ in comparison to control cells (Figure 5A). The effect of recombinant AIF-1 on chemokine secretion by lung fibroblasts was examined in vitro. Cultured lung fibroblasts were stimulated for $24 \mathrm{~h}$ with various concentrations of recombinant AIF-1 or LPS. The level of KC secreted by AIF-1 (10 ng/mL-1 $\mu \mathrm{g} / \mathrm{mL})$-treated lung fibroblasts was significantly higher than that secreted by control cells, and this effect was dose-dependent (Figure 5B). Recombinant AIF-1 did not stimulate the secretion of $\mathrm{KC}$ from RAW264.7 cells (data not shown).

\section{Discussion}

The current study has shown that AIF-1 was expressed in lung macrophages and increased in BALF from mice with bleomycin-induced ALI. This study also showed that recombinant AIF-1 stimulated the secretion of IL-6 and TNF- $\alpha$ in RAW264.7 cells and lung fibroblasts and it stimulated the secretion of $\mathrm{KC}$ in lung fibroblasts.
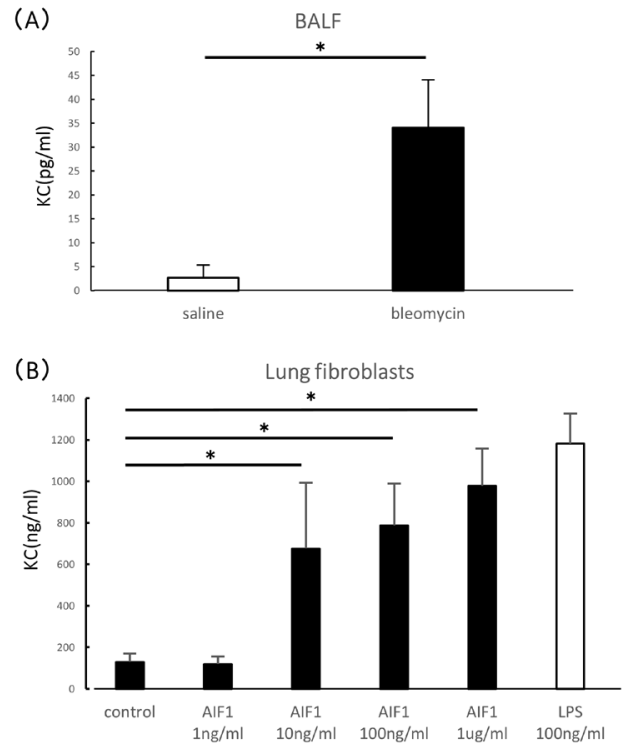

Figure 5. The level of KC in BALF from mice with bleomycin-induced acute lung injury and the level of $\mathrm{KC}$ secreted from lung fibroblasts stimulated by recombinant AIF-1. (A) KC level in BALF ( $n=4$ mice per group). BALF analyses were performed on Day 1 after intratracheal administration of bleomycin. (B) KC level in supernatant $(n=$ 5 mice per group). The concentration of $\mathrm{KC}$ in the supernatant from cultured lung fibroblasts was measured after cells were exposed to various concentrations of rAIF-1 for $24 \mathrm{~h}$. Each bar represents the mean $\pm \mathrm{SE} .{ }^{*} p<0.05$.

Taken together, these findings suggest that AIF-1 plays an important role in the mechanism underlying the etiology of ALI.

AIF-1 was expressed in macrophages in lung tissue with bleomycin-induced ALI. Evidence of AIF1 expression in macrophages coincides with previous studies that detected AIF-1 in macrophages in a model of atherosclerotic allogenic heart grafts or in human macrophage-like cell lines $(13,25)$. AIF-1 was also expressed in BALF from mice with bleomycin-induced ALI. These results suggest that AIF-1 was produced by lung macrophages in bleomycin-induced ALI and that AIF-1 may play a role in the mechanism of inflammation.

Bleomycin induces inflammatory changes in lung tissue with increased expression of pro-inflammatory cytokines in a short period of time (26). In the current study, levels of IL- 6 and TNF- $\alpha$ were significantly increased in BALF from mice exposed to bleomycin in comparison to the control group on Day 1. Both IL-6 and TNF- $\alpha$ are known to be crucial pro-inflammatory cytokines that have variable biological effects on inflammation, immune responses, cell differentiation and proliferation, hematopoiesis, and oncogenesis (27-29). The current study also indicated that recombinant AIF1 stimulates the production of IL- 6 and TNF- $\alpha$ from a macrophage cell line (RAW264.7) and lung fibroblasts in vitro. Although the biological function of lung macrophages has not been completely elucidated and additional research is needed to clarify the role that AIF- 
1 plays, the current results suggest that AIF-1 is produced by lung macrophages and that lung macrophages might produce IL- 6 and TNF- $\alpha$ as autocrines.

A study has reported that fibroproliferative activity is present in the early phase in lungs of patients with ARDS (30). Histology has also indicated that fibroproliferation is present in the early phase in the lungs of patients with ARDS $(31,32)$. As indicated above, lung fibroblasts are thought to play an important role in ALI. Previous studies by the current authors demonstrated that recombinant AIF-1 can stimulate the secretion of IL-6 in human synoviocytes and normal human dermal fibroblasts $(16,33)$. However, lung fibroblasts secreted TNF- $\alpha$ in addition to IL- 6 when stimulated with recombinant AIF-1. These results suggest that AIF-1 produced by lung macrophages contributes to the development of bleomycin-induced ALI by inducing the production of IL- 6 and TNF- $\alpha$ by stimulating lung macrophages in an autocrinal manner and by stimulating lung fibroblasts in a paracrinal manner.

Another interesting finding from the current study is that the expression of $\mathrm{KC}$ and the number of neutrophils were significantly increased in BALF from mice exposed to bleomycin in comparison to the control group. $\mathrm{KC}$ (CXCL1) has been proposed as a functional homologue of human IL-8 and is associated with neutrophil recruitment and inflammation (34). The level of IL-8 in BALF is reported to be significantly higher in patients who subsequently develop ARDS than in patient who do not develop ARDS $(35,36)$. Ma et al. showed that the level of KC in BALF was elevated on Day 1 after intratracheal administration of bleomycin (37). These results coincide with the current finding. Moreover, the current study also indicated that recombinant AIF-1 stimulated the secretion of $\mathrm{KC}$ in lung fibroblasts. These results suggest that AIF-1 contributes to the recruitment of neutrophils by inducing the production of $\mathrm{KC}$ by lung fibroblasts in bleomycininduced ALI.

In conclusion, the current study showed that AIF-1 was overexpressed in lung macrophages and increased in BALF from mice with bleomycin-induced ALI. AIF-1 can stimulate the secretion of IL- 6 and TNF- $\alpha$ in macrophages and lung fibroblasts. This study also indicated that $\mathrm{KC}$, a chemokine that induces neutrophil recruitment and inflammation, is secreted in lung fibroblasts stimulated with AIF-1. These findings suggest that AIF-1 plays a critical role in the mechanism underlying the etiology of ALI.

\section{Acknowledgements}

We wish to thank Naoko Kajitani and Yukiko Shibayama for their assistance in preparing this manuscript. All authors have declared that there are no financial conflicts of interest in regards to this manuscript.

\section{References}

1. Dushianthan A, Grocott MP, Postle AD, Cusack R. Acute respiratory distress syndrome and acute lung injury. Postgrad Med J. 2011; 87:612-622.

2. Saguil A, Fargo M. Acute respiratory distress syndrome: Diagnosis and management. Am Fam Physician. 2012; 85:352-358.

3. Martin TR. Lung cytokines and ARDS: Roger S. Mitchell Lecture. Chest. 1999; 116:2S-8S.

4. Parsons PE, Eisner MD, Thompson BT, Matthay MA, Ancukiewicz M, Bernard GR, Wheeler AP; NHLBI Acute Respiratory Distress Syndrome Clinical Trials Network. Lower tidal volume ventilation and plasma cytokine markers of inflammation in patients with acute lung injury. Crit Care Med. 2005; 33:1-6; discussion 230232.

5. Piantadosi CA, Schwartz DA. The acute respiratory distress syndrome. Ann Intern Med. 2004; 141 :460-470.

6. Windsor AC, Mullen PG, Fowler AA, Sugerman HJ. Role of the neutrophil in adult respiratory distress syndrome. Br J Surg. 1993; 80:10-17.

7. Matthay MA, Ware LB, Zimmerman GA. The acute respiratory distress syndrome. J Clin Invest. 2012; 122:2731-2740.

8. Matthay MA, Ware LB, Zimmerman Matthay MA, Zemans RL. The acute respiratory distress syndrome: Pathogenesis and treatment. Annu Rev Pathol. 2011; 6:147-163.

9. Matute-Bello G, Frevert CW, Martin TR. Animal models of acute lung injury. Am J Physiol Lung Cell Mol Physiol. 2008; 295:L379-399.

10. Autieri MV. cDNA cloning of human allograft inflammatory factor-1: Tissue distribution, cytokine induction, and mRNA expression in injured rat carotid arteries. Biochem Biophys Res Commun. 1996; 228:2937.

11. Deininger MH, Seid K, Engel S, Meyermann R, Schluesener HJ. Allograft inflammatory factor-1 defines a distinct subset of infiltrating macrophages/microglial cells in rat and human gliomas. Acta Neuropathol. 2000; 100:673-680.

12. Iris FJ1, Bougueleret L, Prieur S, Caterina D, Primas G, Perrot V, Jurka J, Rodriguez-Tome P, Claverie JM, Dausset J, Cohen D. Dense Alu clustering and a potential new member of the NF kappa B family within a 90 kilobase HLA class III segment. Nat Genet. 1993; 3:137145.

13. Utans U, Liang P, Wyner LR, Karnovsky MJ, Russell ME. Chronic cardiac rejection: Identification of five upregulated genes in transplanted hearts by differential mRNA display. Proc Natl Acad Sci U S A. 1994; 91:6463-6467.

14. Utans U, Arceci RJ, Yamashita Y, Russell ME. Cloning and characterization of allograft inflammatory factor-1: A novel macrophage factor identified in rat cardiac allografts with chronic rejection. J Clin Invest. 1995; 95:2954-2962.

15. Hara H, Ohta M, Ohta K, Nishimura M, Obayashi H, Adachi T. Isolation of two novel alternative splicing variants of allograft inflammatory factor-1. Biol Chem. 1999; 380:1333-1336.

16. Kimura M, Kawahito Y, Obayashi H, Ohta M, Hara H, Adachi T, Tokunaga D, Hojo T, Hamaguchi M, Omoto A, 
Ishino H, Wada M, Kohno M, Tsubouchi Y, Yoshikawa T. A critical role for allograft inflammatory factor-1 in the pathogenesis of rheumatoid arthritis. J Immunol. 2007; 178:3316-3322.

17. Nagakawa Y, Nomoto S, Kato Y, Montgomery RA, Williams GM, Klein AS, Sun Z. Over-expression of AIF-1 in liver allografts and peripheral blood correlates with acute rejection after transplantation in rats. Am J Transplant. 2004; 4:1949-1957.

18. Morohashi T, Iwabuchi K, Watano K, Dashtsoodol N, Mishima T, Nakai Y, Shimada S, Nishida R, Fujii $\mathrm{S}$, Onoé K. Allograft inflammatory factor-1 regulates trinitrobenzene sulphonic acid-induced colitis. Immunology. 2003; 110:112-119.

19. Zhou X, He Z, Henegar J, Allen B, Bigler S. Expression of allograft inflammatory factor-1 (AIF-1) in acute cellular rejection of cardiac allografts. Cardiovasc Pathol. 2011; 20:e177-184.

20. Yang ZF, Ho DW, Lau CK, Lam CT, Lum CT, Poon RT, Fan ST. Allograft inflammatory factor-1 (AIF-1) is crucial for the survival and pro-inflammatory activity of macrophages. Int Immunol. 2005; 17:1391-1397.

21. Watano K, Iwabuchi K, Fujii S, Ishimori N, Mitsuhashi S, Ato M, Kitabatake A, Onoé K. Allograft inflammatory factor-1 augments production of interleukin-6, -10 and -12 by a mouse macrophage line. Immunology. 2001; 104:307-316.

22. Murakami K, Kohno M, Kadoya M, Nagahara H, Fujii W, Seno T, Yamamoto A, Oda R, Fujiwara H, Kubo T, Morita S, Nakada H, Hla T, Kawahito Y. Knock out of S1P3 receptor signaling attenuates inflammation and fibrosis in bleomycin-induced lung injury mice model. PLoS One. 2014; 9:e106792.

23. Zhou Y, Zhang X, Tan M, Zheng R, Zhao L. The effect of NF-kappaB antisense oligonucleotide on transdifferentiation of fibroblast in lung tissue of mice injured by bleomycin. Mol Biol Rep. 2014; 41:4043-4051.

24. He Z, Deng Y, Li W, Chen Y, Xing S, Zhao X, Ding J, Gao Y, Wang X. Overexpression of PTEN suppresses lipopolysaccharide-induced lung fibroblast proliferation, differentiation and collagen secretion through inhibition of the PI3-K-Akt-GSK3beta pathway. Cell Biosci. 2014; 4:2.

25. Utans U, Quist WC, McManus BM, Wilson JE, Arceci RJ, Wallace AF, Russell ME. Allograft inflammatory factory-1. A cytokine-responsive macrophage molecule expressed in transplanted human hearts. Transplantation. 1996; 61:1387-1392.

26. Moeller A, Ask K, Warburton D, Gauldie J, Kolb M. The bleomycin animal model: A useful tool to investigate treatment options for idiopathic pulmonary fibrosis? Int $\mathrm{J}$ Biochem Cell Biol. 2008; 40:362-382.
27. Ataie-Kachoie P, Pourgholami MH, Richardson DR, Morris DL. Morris, Gene of the month: Interleukin 6 (IL6). J Clin Pathol. 2014; 67:932-937.

28. Mukhopadhyay S, Hoidal JR, Mukherjee TK. Role of TNFalpha in pulmonary pathophysiology. Respir Res. 2006; 7:125.

29. Simpson SQ, Casey LC. Role of tumor necrosis factor in sepsis and acute lung injury. Crit Care Clin. 1989; 5:27-47.

30. Burnham EL, Janssen WJ, Riches DW, Moss M, Downey GP. The fibroproliferative response in acute respiratory distress syndrome: Mechanisms and clinical significance. Eur Respir J. 2014; 43:276-285.

31. Bulpa PA, Dive AM, Mertens L, Delos MA, Jamart J, Evrard PA, Gonzalez MR, Installé EJ. Combined bronchoalveolar lavage and transbronchial lung biopsy: Safety and yield in ventilated patients. Eur Respir J. 2003; 21:489-494.

32. Papazian L, Doddoli C, Chetaille B, Gernez Y, Thirion X, Roch A, Donati Y, Bonnety M, Zandotti C, Thomas P. A contributive result of open-lung biopsy improves survival in acute respiratory distress syndrome patients. Crit Care Med. 2007; 35:755-762.

33. Yamamoto A, Ashihara E, Nakagawa Y, Obayashi H, Ohta M, Hara H, Adachi T, Seno T, Kadoya M, Hamaguchi M, Ishino H, Kohno M, Maekawa T, Kawahito Y. Allograft inflammatory factor- 1 is overexpressed and induces fibroblast chemotaxis in the skin of sclerodermatous GVHD in a murine model. Immunol Lett. 2011; 135:144150.

34. Rubio N, Sanz-Rodriguez F. Induction of the CXCL1 (KC) chemokine in mouse astrocytes by infection with the murine encephalomyelitis virus of Theiler. Virology. 2007; 358:98-108.

35. Donnelly SC, Strieter RM, Kunkel SL, Walz A, Robertson CR, Carter DC, Grant IS, Pollok AJ, Haslett C. Interleukin- 8 and development of adult respiratory distress syndrome in at-risk patient groups. Lancet. 1993; 341:643-647.

36. Jorens PG, Van Damme J, De Backer W, Bossaert L, De Jongh RF, Herman AG, Rampart M. Interleukin 8 (IL-8) in the bronchoalveolar lavage fluid from patients with the adult respiratory distress syndrome (ARDS) and patients at risk for ARDS. Cytokine. 1992; 4:592-597.

37. Ma L, Shaver CM, Grove BS, Mitchell DB, Wickersham NE, Carnahan RH, Cooper TL, Brake BE, Ware LB, Bastarache JA. Kinetics of lung tissue factor expression and procoagulant activity in bleomycin induced acute lung injury. Clin Trans1 Med. 2015; 4:63.

(Received February 16, 2016; Accepted February 19, 2016) 